\title{
Circulating nucleosomes as new blood-based biomarkers for detection of colorectal cancer
}

\author{
Jean-François Rahier ${ }^{1 *}$, Anne Druez ${ }^{1}$, Laurence Faugeras², Jean-Paul Martinet', Myriam Géhénot, \\ Eléonore Josseaux ${ }^{3}$, Marielle Herzog ${ }^{3}$, Jake Micallef ${ }^{3}$, Fabienne George ${ }^{4}$, Monique Delos ${ }^{5}$, Thierry De Ronde ${ }^{1}$, \\ Abdenor Badaoui ${ }^{1}$ and Lionel D'Hondt ${ }^{2}$
}

\begin{abstract}
Background: Colonoscopy is currently widely accepted as the gold standard for detection of colorectal cancer (CRC) providing detection of up to $95 \%$ of pre-cancerous lesions during the procedure. However, certain limitations exist in most countries including cost and access to the procedure. Moreover, colonoscopy is an invasive technique with risk inherent to the endoscopic procedure. For this reason, alternative screening tests, in particular, fecal occult blood-based tests, have been widely adopted for frontline screening. Limited compliance to colonoscopy and fecal screening approaches has prompted research on blood-based tests as an alternative approach to identifying individuals at risk who could then be referred for colonoscopy. Increased total levels of nucleosomes in the blood have been associated with tumor burden and malignancy progression. Here, we report for the first time, CRC-associated epigenetic profiles of circulating cell-free nucleosomes (cf-nucleosomes).
\end{abstract}

Methods: Levels of 12 epigenetic cf-nucleosome epitopes were measured in the sera of 58 individuals referred for endoscopic screening for CRC.

Results: Multivariate analysis defined an age-adjusted panel of four cf-nucleosomes that provided an AUC of 0.97 for the discrimination of CRC from healthy controls with high sensitivity at early stages (sensitivity of 75 and 86 at 90\% specificity for stages I and II, respectively). A second combination of four cf-nucleosome biomarkers provided an AUC of 0.72 for the discrimination of polyps from the healthy group.

Conclusions: This study suggests that a combination of different cf-nucleosome structures analyzed in serum samples by a simple ELISA is a promising approach to identify patients at risk of CRC.

Keywords: Nucleosomes, Colorectal cancer, Epigenetics, Blood-based screening test

\section{Background}

Colorectal Cancer (CRC) is the third most common cancer worldwide with approximately 1.36 million new cases/year and nearly 700,000 CRC related deaths every year [1]. Early detection of CRC significantly improves patient outcome and is a key factor in reducing mortality [2]. Screening programs, such as fecal occult blood testing (FOBT) or fecal immunochemical testing (FIT),

\footnotetext{
* Correspondence: jrahier@gmail.com

'CHU UCL Namur, Service d'Hépato-gastroentérologie, Université catholique de Louvain, Av. Docteur G. Thérasse 1, B-5530 Yvoir, Belgium

Full list of author information is available at the end of the article
}

colonoscopy, and sigmoidoscopy, have led to improved CRC detection [3-5] but CRC screening is still under-used and participation in the USA rarely reaches $65 \%$ of the target population [6]. A bloodbased, minimally invasive test is seen as a highly attractive approach to increase screening compliance and CRC detection. Moreover, in the majority of cases, the disease develops over many years through the so-called adenoma (polyp)-carcinoma sequence. Therefore, it is widely accepted that detection and removal of pre-cancerous lesions can prevent progression to cancer $[7,8]$. 
Over the last decade, several studies have shown that alteration of epigenetic marks, including DNA methylation, and anomalous post-transcriptional modification of histones are hallmarks of cancers. Genome-wide epigenetic signals have been shown to be altered in cancer cells, and accumulating evidence indicates that these epigenetic changes occur early in tumorogenesis [9]. In the nucleus of eukaryotic cells, DNA is structured with eight histone proteins in nucleosomes. Further compaction by linker histone $\mathrm{H} 1$ condenses the nucleosomes into chromatin and ultimately into chromosomes. Increased levels of nucleosomes in the blood following cell death and fragmentation have been associated with tumor burden and malignant progression in several types of cancers [10-12]. However, the diagnostic value of nucleosomes is limited since various benign diseases associated with accelerated cell death such as degenerative diseases, autoimmune disease, ischemia, or trauma are also associated with an elevated circulating cell-free levels of nucleosomes [10]. Physiologically, nucleosomes can also be released by immune system cells. These strands of decondensed chromatin with associated myeloperoxidase are known as neutrophil extracellular traps and form part of the innate response to pathogens in a process called NETosis [13, 14]. The most studied potential epigenetic circulating cellfree DNA (ccfDNA) biomarkers in cancer are the methylation levels of a variety of tumor suppressor genes, particularly septin-9 gene that show promising results in CRC detection [15-17].

Studies on global dysregulation of epigenetic markers on circulating nucleosomes, such as DNAmethylation and histone modifications have been also associated with colorectal or pancreatic cancer [18-20]. Changes in histone modification patterns detected on circulating nucleosomes could therefore be powerful blood-based biomarkers enabling early cancer detection.

Using a novel ELISA platform-Nucleosomics ${ }^{\circ}$ Belgian Volition, we evaluated the capacity of blood-basedspecific epigenetic features of circulating nucleosomes to detect colorectal cancer. We report the ability of global epigenetic profiling in circulating cell-free nucleosomes (cf-nucleosomes) to distinguish colorectal cancer and pre-cancerous lesions (polyps) from healthy controls.

\section{Methods}

\section{Patients}

This study included 58 patients over 50 years of age referred to the endoscopic unit of the University hospital CHU UCL Namur for colonic surveillance or secondary to bowel symptoms. The study, approved by the Ethic Committee of the CHU UCL Namur (Reference number EC: $95 / 2011)$ and declared to the Belgian authorities $\left(\mathrm{N}^{\circ}\right.$ BO39201112452), was conducted between October 2012 and March 2015. All patients gave informed consent to participate to the study. Blood samples were obtained during outpatient consultations prior to the diagnostic colonoscopy. Patients were classified into three groups based on their colonoscopy reports: (i) patients with CRC ( $n=23)$, (ii) patients with polyps $(n=16)$, and (iii) healthy controls with no endoscopic lesions $(n=19)$. Patients with ongoing/previous history of cancer within the last 5 years, or with a diagnosis of inflammatory bowel disease and/or with infectious disease within 6 weeks were not included in the study.

\section{Serum samples}

Blood samples were processed, clinically annotated, anonymized, and aliquoted at the Biobank of the CHU UCL Namur. Whole-blood samples were collected by venipuncture using Venosafe Plastic Tubes (product $\mathrm{n}^{\circ}$ VF-109SP, Terumo Europe). Clotting time was $30 \mathrm{~min}$ after which the samples were centrifuged at $3000 \mathrm{~g}$ for $15 \mathrm{~min}$ at $4{ }^{\circ} \mathrm{C}$ and the serum fraction collected. Ten millimolar EDTA ( $\mathrm{pH}$ 8) was added to stabilize the nucleosomes in the serum which were aliquoted, frozen, and stored at $-80{ }^{\circ} \mathrm{C}$.

\section{Circulating cf-nucleosome ELISA}

Twelve epitopes on circulating cf-nucleosomes were measured using specific ELISA assays $\left(\mathrm{NuQ}^{\circ}\right.$, Belgian Volition SPRL, Namur, Belgium) performed according to manufacturer instructions, as previously reported [18]. The epigenetics markers were chosen based on published evidence of their role in cancer and availability of well-validated assays. Briefly, serum samples $(10 \mu \mathrm{l}$ in duplicate) were incubated in 96-well plates coated with monoclonal nucleosome capture antibody. Following a wash step, the samples were incubated with biotinylated antibodies, raised against the specific nucleosome epitopes. After a second wash step, a streptavidinhorseradish peroxidase (HRP) conjugate was added and incubated for $30 \mathrm{~min}$. Peroxidase substrate (2-2'-azinobis(3-ethylbenzothiazoline-6-sulfonic) was added, and the optical densities of the wells were read after $20 \mathrm{~min}$ with an X-Mark Microplate spectrophotometer. The specific nucleosome epitopes analyzed included nucleosomeassociated histone modifications: H4K20me3 (mAb), H4PanAc (mAb), pH2AX (mAb), H3K9Me3 (pAb), H2AK119Ub (mAb), H3K9Ac (mAb), or H3K27Ac $(\mathrm{mAb})$; nucleosome-associated DNA modification: $5 \mathrm{mC}$ $(\mathrm{mAb})$; nucleosome containing histone variants: $\mathrm{H} 2 \mathrm{AZ}$ $(\mathrm{mAb})$; nucleosome-protein adducts: HMGB1 (mAb) and EZH2 (mAb); and a conserved nucleosome epitope as a measure of total nucleosomes.

The performed measurements of the levels of cfnucleosomes were expressed qualitatively in the output from the ELISA detection as optical density (OD). All ELISA measurements on each serum sample were performed in 
duplicate, and the results used for the statistical analysis were expressed as the mean of the duplicate measurement.

In order to minimize inter-assay bias, CRC patient samples, patient polyp samples, and control samples were randomized in all plates and $\mathrm{QC}$ samples were used over all plates.

\section{CEA}

The level of the carcinoembryonic antigen (CEA) serum marker was evaluated using a commercial CEA ELISA kit (RE59101, IBL international) according the manufacturer instructions.

\section{Statistical analysis}

The analysis was conducted using the statistical programming language $R$ [21]. Samples were assigned to three groups: healthy, cancer, or benign. The data were pre-processed, taking the logarithm to base 2 for each of the values for the $12 \mathrm{NuQ}^{\circ}$ assays and CEA, subtracting the mean for the assay, and dividing by the standard deviation for each assay. Linear multivariate models were calculated using Fisher's linear discriminant analysis. The best models from the comparisons of CRC vs. healthy (algorithm 1) and polyps vs. healthy (algorithm 2) were selected. An upper limit of five variables was imposed to avoid overtraining. This cut-off of five variables was estimated based on the calculation of the RTMSPE-the root trimmed mean square predictor error-for each panel size alongside its performance.

\section{Results}

\section{Patients}

The demographics and the clinical characteristics of patients and lesions are shown in Tables 1, 2, and 3. The cancer group included four patients at stage $0-\mathrm{I}$, seven patients at stage II, seven patients at stage III, and five patients at stage IV. Most of the tumors were of intermediate and high grade (21 out of 23). Cancer staging and work-up was done according to NCCN guidelines https://www.nccn.org/professionals/physician_gls/pdf/
Table 2 Clinical characteristics of the colorectal cancer in 23 patients

\begin{tabular}{|c|c|c|}
\hline Clinical stage & Tumor localisation & Tumor grade \\
\hline 0 & C20.9 & $\|$ \\
\hline 0 & C20.9 & $\|$ \\
\hline I & C20.9 & III \\
\hline । & C20.9 & III \\
\hline$\| \mathrm{A}$ & C18.2 & III \\
\hline$\| \mathrm{A}$ & C18.2; C18.4 & III \\
\hline$\| A$ & C18.2 & $\|$ \\
\hline$\| \mathrm{A}$ & C18.7 & $\|$ \\
\hline$\| A$ & C20.9 & III \\
\hline$\| C$ & C18.7 & $\|$ \\
\hline$\| C$ & C18.2 & 1 \\
\hline III B & C18.7 & $\|$ \\
\hline III B & C18.2 & III \\
\hline III B & C18.6 & $\|$ \\
\hline III B & C18.2 & $\|$ \\
\hline III B & C18.2 & । \\
\hline III B & C18.2 & III \\
\hline III C & C18.6 & $\|$ \\
\hline IV & C18.7 & III \\
\hline IV & C18.7 & $\|$ \\
\hline IV & C18.2; C20.9 & $\|$ \\
\hline IV & C18.2; C20.9 & $\|$ \\
\hline IV & C18.2 & $\|$ \\
\hline
\end{tabular}

colon.pdf and https://www.nccn.org/professionals/physician_gls/pdf/rectal.pdf. The polyp group included 51 lesions in 16 patients. Ten patients with dysplastic polyps and six patients with hyperplastic polyps.

\section{Serum analysis}

Univariate analysis

The epigenetic profiles of the circulating cf-nucleosomes of the 58 subjects were investigated using 12 separate

Table 1 Demographics of the patient population

\begin{tabular}{lllll}
\hline Diagnosis & Patients $(n)$ & Age (median, IQR) & Male:female & Smoking:non-smoking \\
\hline CRC & 23 & $79(70-83)$ & $16: 7$ & $2: 21$ \\
Stage 0-I & 4 & $70(65-75)$ & $3: 1$ & $0: 4$ \\
Stage II & 7 & $80(74-83)$ & $4: 3$ & $1: 6$ \\
Stage III & 7 & $81(72-82)$ & $6: 1$ & $1: 6$ \\
Stage IV & 5 & $80(78-82)$ & $3: 2$ & $0: 5$ \\
Polyp & 16 & $65(56-67)$ & $10: 6$ & $7: 9$ \\
Hyperplastic & 6 & $60(53-64)$ & $1: 5$ & $2: 4$ \\
Dysplastic & 10 & $66(60-67)$ & $9: 1$ & $5: 5$ \\
Healthy & 19 & $62(58-66)$ & $11: 9$ & $2: 17$ \\
\hline
\end{tabular}


Table 3 Histological and morphologic characteristics of 51 polyps in 16 patients

\begin{tabular}{lll}
\hline $\begin{array}{l}\text { Histologic classification } \\
(N=51)\end{array}$ & $\begin{array}{l}\text { Number of polyps/ } \\
\text { patient (median, IQR) }\end{array}$ & $\begin{array}{l}\text { Size of polyps } \\
(\mathrm{mm}) \text { (median, IQR) }\end{array}$ \\
\hline $\begin{array}{l}\text { Hyperplastic and } \\
\text { sessile serrated }\end{array}$ & $1(1-2.75)$ & $5(5-5.25)$ \\
$N=28$ & \\
$\begin{array}{l}\text { Adenomatous low } \\
\text { grade dysplasia }\end{array}$ & $1(1-3)$ & $9(6-10)$ \\
$N=21$ & & $25(17.5-32.5)$ \\
$\begin{array}{l}\text { Adenomatous } \\
\text { high-grade dysplasia }\end{array}$ & 1 & \\
$N=2$ & & \\
\hline
\end{tabular}

$\mathrm{NuQ}^{\odot}$ ELISA assays. The areas under the ROC (receiver operating characteristics) curve (AUCs) for individual cfnucleosome biomarkers varied from 0.51 to 0.76 for the discrimination of the cancer vs. healthy groups with diagnostic sensitivities (at 90\% specificity) varying from 0 to $39 \%$ (Table 4). In our study, the established tumor marker carcinoembryonic antigen (CEA) provided a sensitivity of $35 \%$ at $90 \%$ specificity with an AUC of 0.66 .

\section{Multivariate analysis}

In order to improve clinical performance, we evaluated the cumulative performance of cf-nucleosome biomarkers alone or in combination with CEA and adjusted for age using multivariate analysis. Linear models, based on a weighted sum of one to five variables (restricted to avoid overtraining), were developed using Fisher's linear discriminant analysis optimized for AUC. For discrimination between colorectal cancer cases and healthy subjects, a four-cf-nucleosome biomarker combination was selected (see Methods, algorithm 1) utilizing histone modifications

Table 4 Epigenetic profiles of circulating nucleosomes, AUC, and sensitivity at $90 \%$ specificity

\begin{tabular}{lll}
\hline & \multicolumn{2}{l}{ Cancer vs. healthy } \\
\cline { 2 - 3 } & AUC & Sensitivity at 90\% specificity \\
\hline H2AK119Ub & 0.76 & $39 \%$ \\
H3K9me3 & 0.65 & $17 \%$ \\
EZH2 & 0.66 & $0 \%$ \\
H3K9AC & 0.58 & $13 \%$ \\
H3K27AC & 0.58 & $0 \%$ \\
H4pan Ac & 0.57 & $0 \%$ \\
pH2AX & 0.57 & $0 \%$ \\
5mC & 0.55 & $9 \%$ \\
H4K20me3 & 0.53 & $0 \%$ \\
HMGB1 & 0.53 & $0 \%$ \\
Nucleosome & 0.53 & $0 \%$ \\
H2AZ & 0.51 & $0 \%$ \\
CEA & 0.66 & $35 \%$ \\
\hline
\end{tabular}

H2AK119Ub, H3K9Ac, H3K27Ac, and the global level of nucleosomes. The combination of these four cfnucleosome biomarkers increased sensitivity for detection of CRC to $74 \%$ at $90 \%$ specificity compared with the best single assay sensitivity of $39 \%$. Significant separation was achieved between the CRC and the healthy groups as shown in the box plot (median 0.212 vs. $-0.494, p<0.001$ ) (Fig. 1). The AUC for the discrimination of cancer vs. healthy was 0.87 - a significant improvement compared with CEA alone (Fig. 2). It should also be noted that while this combination of four cf-nucleosome biomarkers did not enable discrimination between healthy controls and polyps, it did show a statistical significant separation between the CRC and the polyp group $(p=0.006)$.

Increasing to a five-cf-nucleosome biomarker panel from the panel of 12 selected did not further improve sensitivity (data not shown). However, combining the four-nucleosome biomarker panel with CEA testing provided a moderate increase in sensitivity (at $90 \%$ specificity) to $78 \%$ for CRC vs. that of the healthy controls (Fig. 2).

The nucleosome biomarkers were shown to be independent of age with only a marginal coefficient of correlation with patient-derived physiological parameters including age and gender. The Pearson correlation coefficient ranged between -0.28 and 0.23 (Additional file 1: Table S1). The score from the age-adjusted four-cf-nucleosome biomarker algorithm was significantly higher $(p<0.001)$ in the sera of patients with colorectal cancer compared with the healthy

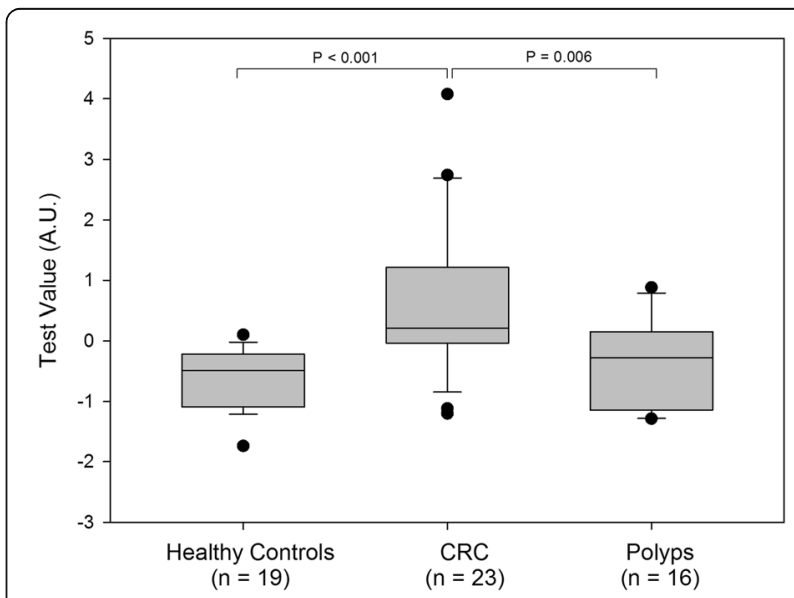

Fig. 1 Combination of four cf-nucleosome biomarkers in CRC patients, patients with polyps, and healthy controls. Box plot demonstrating significantly higher score in patients with a CRC $(n=23)$ compared with healthy controls $(n=19)(p<0.001)$ and between CRC patients $(n=23)$ and patients with polyps $(n=16)(p=0.006)$. The score for each group was achieved with pre-processed NuQ $Q^{\bullet}$ ELISA data from four cf-nucleosome biomarkers: histone modifications H2AK119Ub, H3K9AC, H3K27Ac, and the global level of nucleosome. Fisher's linear discriminant model was used to calculate the score. $p$ values were determined by Mann-Whitney rank-sum test. The box plot shows the median and the 25th and 75th percentiles; the whiskers indicate the 5th and 95th percentiles. A.U. arbitrary unit 


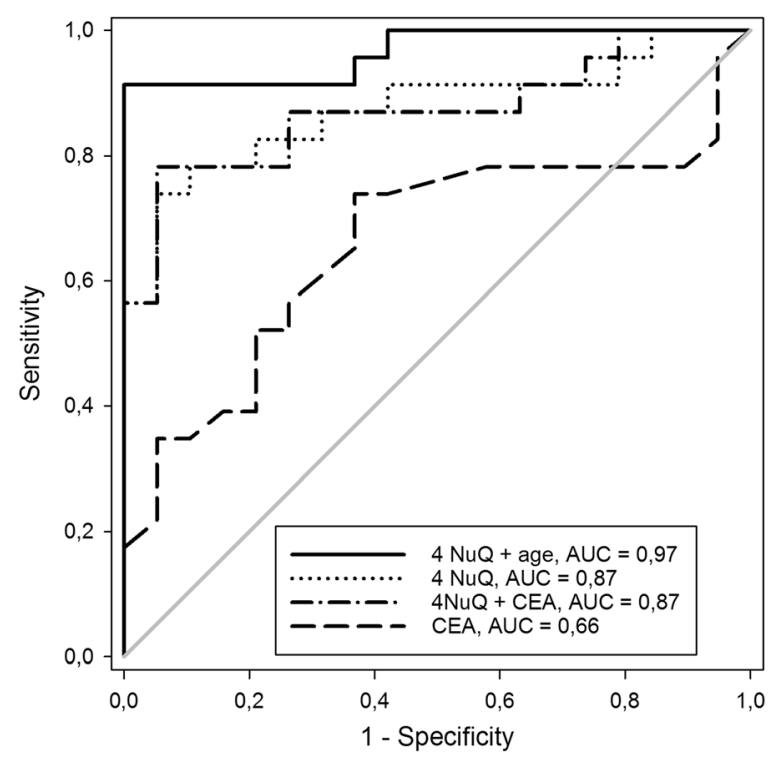

Fig. 2 ROC curves for discrimination of cancer vs. healthy controls. The area under the curve $(A \cup C)$ for the established tumor marker CEA (0.66) was improved by using the best combination of four cf-nucleosome biomarkers (0.87); or the panel of four-cf-nucleosome biomarker panel with CEA (0.87). The AUC was further increased by using the age-adjusted four-cf-nucleosome panel (0.97); the gray line indicates random chance

controls and the polyp group (median 1.49 vs. -1.39 and -1.04) (Fig. 3), and the AUC increased to 0.97 with a diagnostic sensitivity of $91 \%$ at $90 \%$ specificity (Fig. 2 ).

These results exceeded the performance of the established tumor marker CEA. CEA is generally recommended for the follow-up of CRC patients after treatment but not for diagnosis because of its poor sensitivity for detection of early stage disease [22]. In the present study, the single biomarker CEA showed relatively good sensitivity in stage IV ( $75 \%$ sensitivity at $90 \%$ specificity) but, as expected, performed poorly in the earliest stages $(0,14$, and 57 sensitivity at $90 \%$ specificity in stages I, II, and III, respectively) (Table 5). Conversely, at the same specificity, the four-cf-nucleosome biomarker panel showed markedly increased sensitivity across all stages of CRC ( $75 \%$ for stage I cancer, $86 \%$ for stage II cancer, $71 \%$ for stage III, and $60 \%$ for stage IV cancer) vs. healthy patients. This increased sensitivity was also observed with the four cfnucleosome biomarkers in the age-adjusted algorithm ( 75 , 86,100 , and $100 \%$ at the respective stages (Table 5).

Importantly, colonoscopy can detect and remove polyps as they may be precursor lesions for most colorectal cancers. Detection of polyps is therefore highly desirable. Applying the first panel to the polyp cohort provided a sensitivity for detection of $31 \%$ (at $90 \%$ specificity) relative to the healthy group-a significant decrease in performance compared with the cancer group (data not shown). A second algorithm, optimized for discrimination between

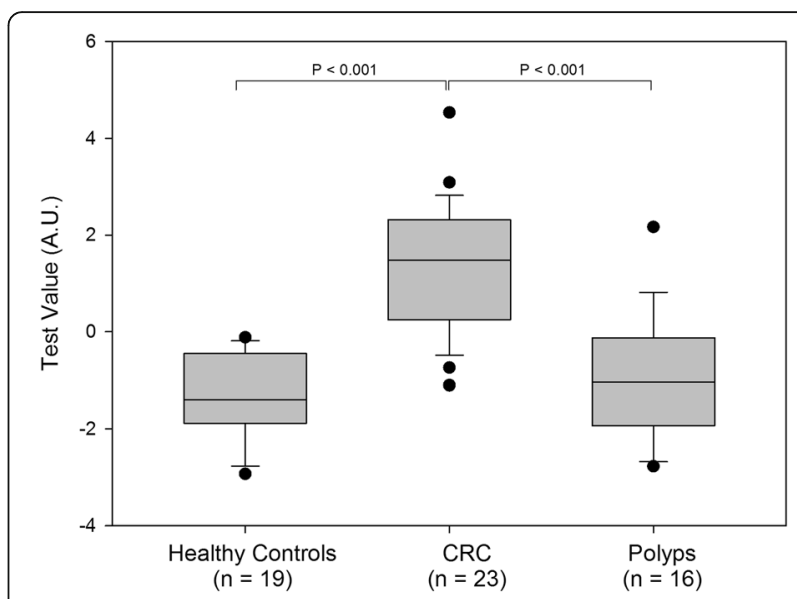

Fig. 3 Discrimination of four $\mathrm{NuQ}^{\circledR}$ assay panels in an age-adjusted algorithm CRC, polyps, and healthy controls. The box plot shows significantly higher scores in patients with a CRC $(n=23)$ compared with healthy controls $(n=19)(p<0.001)$ and between CRC patients $(n=23)$ and patients with polyps $(n=16)(p<0.001)$. The score for each group was performed with pre-processed NuQ ${ }^{\circledR}$ ELISA data from four cf-nucleosome biomarkers: histone modifications H2AK119Ub, H3K9Ac, H3K27Ac, and the global level of nucleosome and age. Fisher's linear discriminant model was used to calculate the score. $p$ values were determined by Mann-Whitney rank-sum test. The box plot shows the median and the 25th and 75th percentiles; the whiskers indicate the 5th and the 95th percentiles. A.U. arbitrary unit

the polyps and healthy groups, was developed (see Methods algorithm 2) utilizing histone modifications H2AK119Ub, H3K9Ac, H4K20me3, and the global level of nucleosomes. The combination of these four cfnucleosome biomarkers significantly improved discrimination of the polyp vs. healthy groups (median 0.084 vs. $-0.002, p=0.025$ ) (Fig. 4). The sensitivity for polyp detection vs. the healthy group was $62 \%$ (at $90 \%$ specificity) with an AUC of 0.72 (Fig. 5). Adjusting for age did not help to improve the discrimination (data not shown).

\section{Discussion}

Global levels of single cf-nucleosome epitopes have limited ability to discriminate between CRC patients and healthy controls (sensitivity at $90 \%$, specificity $0-39 \%$ ). However, performance is significantly increased when used in combination. A four-cf-nucleosome epitope biomarker panel selected from a screening panel of 12 assays had an AUC of 0.87 for the discrimination of patients with CRC from healthy controls. Seventeen out of 23 CRC cancer cases were detected vs. healthy controls with two false positive results (sensitivity of $74 \%$ with $90 \%$ specificity). The cancers were detected in various parts of the colon: right, left, sigmoid colon, and rectum. In six patients, the diagnosis of colorectal cancer was missed. These patients were in different clinical stages: 1 stage I, 1 stage II, 2 stage III, and 2 stage IV, and missed tumors were also located in various 
Table 5 Percentage of sensitivity at 90\% specificity at the different CRC stages for CEA or combinations of $\mathrm{NuQ}^{\oplus}$ biomarkers

\begin{tabular}{llll}
\hline & \multicolumn{3}{l}{ \% Sensitivity at 90\% specificity } \\
\cline { 2 - 4 } CRC & CEA & $\begin{array}{l}\text { Combination of 4 } \\
\text { NuQ }\end{array}$ & $\begin{array}{l}\text { Combination of } 4 \\
\text { NuQ }\end{array}$ \\
\hline All stages & 35 & 74 & 91 \\
Stagsays I & 0 & 75 & 75 \\
Stage II & 14 & 86 & 86 \\
Stage III & 57 & 71 & 100 \\
Stage IV & 60 & 60 & 100
\end{tabular}

parts of the colon. Adding CEA resulted in a marginal increase in sensitivity at $90 \%$ specificity but no overall increase in the AUC. However, including age as a variable enhanced the performance of four cf-nucleosome biomarkers. Twenty-one of the $23 \mathrm{CRC}$ cancer cases were detected vs. the healthy subjects with two false positive results (sensitivity of $91 \%$ with $90 \%$ specificity). Two early stage cancers, 1 stage I and 1 stage IIA cancer, were missed.

Remarkably, the combination of four cf-nucleosome epitopes was able to detect early stage cancer (stages I and II) as well and perhaps even better than late stage cancer (III and IV). This is of particular interest for potential use of cf-nucleosomes as biomarkers to screen for CRC whereas CEA and SEPT9 are associated with advanced cancer. This is particularly true since screening

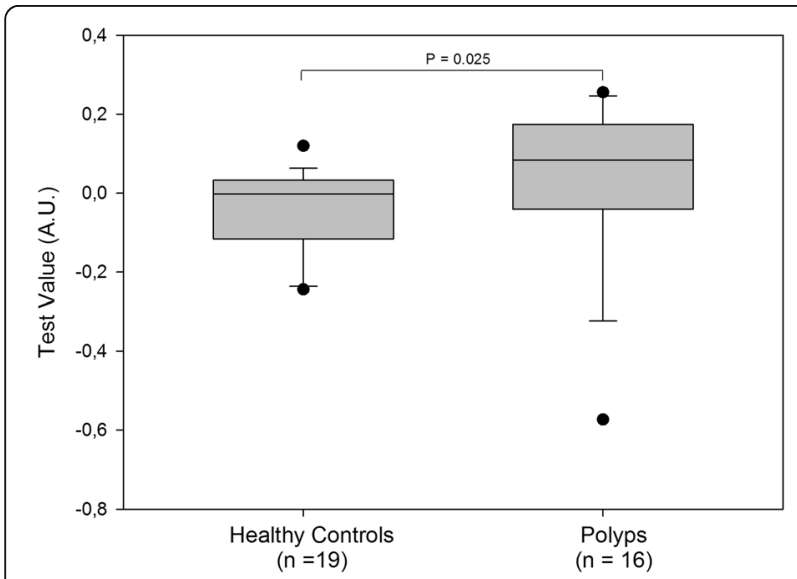

Fig. 4 Combination of four cf-nucleosome biomarkers in polyps vs. healthy controls. The box plot shows significantly higher scores in patients with a polyp $(n=16)$ compared with healthy controls $(n=19)$ ( $p=0.025$ ). Improved discrimination between the polyp and the healthy control groups was achieved using a second algorithm. The score for each group was achieved with pre-processed NuQ ${ }^{\circledR}$ ELISA data from four cf-nucleosome biomarkers: histone modifications H2AK119Ub, H3K9Ac, H4K20Me3, and the global level of nucleosome. Fisher's linear discriminant model was used to calculate the score. $p$ values were determined by Mann-Whitney rank-sum test. The box plot shows the median and the 25th and 75th percentiles; the whiskers indicate the 5 th and the 95th percentiles. A.U. arbitrary unit

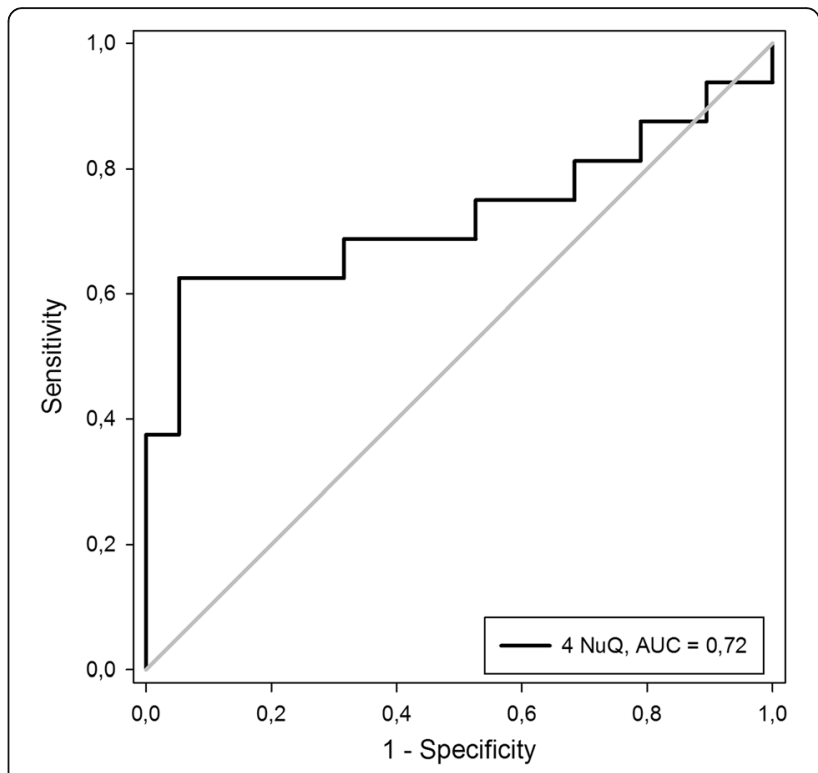

Fig. 5 ROC curve for the combination of four biomarkers distinguishing between patients with polyps and healthy controls. Area under the curve (AUC) 0.72. The gray line indicates random chance

programs mature, and the incidence of later stage cancer decreases. The current study supports the fact that epigenetic changes-epimutations-occur early and could be causative in tumorogenesis. The presence of epimutations on cf-nucleosomes in the blood circulation appears to occur from tumor initiation to advanced stages. The addition of age as a variable increased sensitivity for late-stage cancer but not early stage. Most of our tumors were of high or intermediate grade (21/23). At this point, we cannot establish a relationship between grade and cf-nucleosomes and therefore between the sensitivity of the technique and tumor grade. The relationship between cf-nucleosome levels and clinical evolution or prognosis is currently unknown. However, it will be studied in the near future.

Discrimination of patients with polyps from healthy controls was achieved using a second combination of four $\mathrm{cf}$-nucleosomes (AUC $=0.72$ ). Adding age did not improve this discrimination.

\section{Conclusions}

In conclusion, the clinical performance of the cfnucleosome epitope combination is promising for detection of early stage of CRC and offers a potential non-invasive approach to CRC screening. Based on these encouraging results, we believe that further studies with larger numbers of patients should be performed to confirm and validate the usefulness of cf-nucleosome epigenetic biomarkers in polyps and CRC detection. 


\section{Additional file}

Additional file 1: Table S1. Absence of correlation between $\mathrm{NuQ}^{\oplus}$ assays, age, and gender. Values are expressed in Pearson correlation coefficient. (DOC $30 \mathrm{~kb}$ )

\section{Abbreviation}

A.U.: Arbitrary unit; AUC: Area under the curve; CEA: Carcinoembryonic antigen; Cf-nucleosomes: Cell-free nucleosomes; CRC: Colorectal cancer; DNA: Deoxyribonucleic acid; FIT: Fecal immunochemical testing; FOBT: Fecal occult blood testing; HRP: Streptavidin-horseradish peroxidase; ROC: Receiver operating characteristics

\section{Acknowledgements}

The authors thank Ms Laure Finet (Biobanque CHU UCL Namur) for her excellent technical support.

\section{Fundings}

Nucleosomics ${ }^{\circledast}$ Belgian Volition provided the fundings the for blood sample analysis.

\section{Availability of data and materials}

The datasets during and/or analyzed during the current study are available from the corresponding author on reasonable request.

\section{Authors' contributions}

JFR, TDR, MH, and LD designed the study. JFR, AD, JPM, MG, TDR, and AB performed endoscopies. JFR, LD, AD, and MH collected the data. MD analyzed the histologic lesions. JFR, MH, and LD analyzed the data. JFR, MH, $T D R, A D, E J, M H$, and LD wrote the manuscript. All authors read and approved the final manuscript.

\section{Competing interests}

None of the authors had competing interest except those with affiliation $N^{\circ} 3: E J, M H$ and $J M$

\section{Consent for publication}

Not applicable

\section{Ethics approval and consent to participate}

The study has been approved by the Ethic Committee of the $\mathrm{CHU} \cup \mathrm{CL}$ Namur. All participants provided written informed consent to participate in the study.

\section{Publisher's Note}

Springer Nature remains neutral with regard to jurisdictional claims in published maps and institutional affiliations.

\section{Author details}

${ }^{1} \mathrm{CHU}$ UCL Namur, Service d'Hépato-gastroentérologie, Université catholique de Louvain, Av. Docteur G. Thérasse 1, B-5530 Yvoir, Belgium. ${ }^{2} \mathrm{CHU} \cup C L$ Namur, Service d'Oncologie, Université catholique de Louvain, Av. Docteur G. Thérasse 1, B-5530 Yvoir, Belgium. ${ }^{3}$ Belgian Volition SPRL, Rue du Séminaire 20A, B-5000 Namur, Belgium. ${ }^{4} \mathrm{CHU}$ UCL Namur, Biobanque, Université catholique de Louvain, Av. Docteur G. Thérasse 1, B-5530 Yvoir, Belgium. ${ }^{5} \mathrm{CHU}$ UCL Namur, Service d'anatomie Pathologique, Université catholique de Louvain, Av. Docteur G. Thérasse 1, B-5530 Yvoir, Belgium.

Received: 16 February 2017 Accepted: 3 May 2017

Published online: 15 May 2017

\section{References}

1. Ferlay J, Soerjomataram I, Dikshit R, et al. Cancer Incidence and mortality worldwide: sources, methods and major patterns in GLOBOCAN 2012. Int Cancer. 2015;136(5):359-86.

2. DeSantis $C E$, Chieh Lin $C$, Mariotto $A B$, et al. Cancer treatment and survivorship statistics, 2014. CA Cancer J Clin. 2014;64:252-71.
3. Hewitson P, Glasziou P, Watson E, Towler B, Irwig L. Cochrane systematic review of colorectal cancer screening using the fecal occult blood test (Hemoccult): an update. Am J Gastroenterol. 2008;103(6):1541-9.

4. Brenner $\mathrm{H}$, Chang-Claude J, Jansen L, Knebel P, Stock C, Hoffmeister M. Reduced risk of colorectal cancer up to 10 years after screening, surveillance, or diagnostic colonoscopy. Gastroenterology. 2014;146(3):709-17.

5. Schreuders EH, Ruco A, Rabeneck L, et al. Colorectal cancer screening: a global overview of existing programmes. Gut. 2015;64(10):1637-49.

6. Winawer SJ, Fischer SE, Levin B. Evidence-based, reality-driven colorectal cancer screening guidelines: the critical relationship of adherence to effectiveness. JAMA. 2016:315(19):2065-6.

7. Brenner H, Kloor M, Pox CP. Colorectal cancer. Lancet. 2014;383:1490-502.

8. Kedrin D, Gala MK. Genetics of the serrated pathway to colorectal cancer. Clin Transl Gastroenterol. 2015;6(4):84.

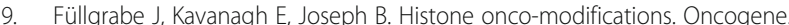
2011;30(31):3391-403.

10. Holdenrieder S, Stieber $\mathrm{P}$, Bodenmüller $\mathrm{H}$, et al. Circulating nucleosomes in serum. Ann N Y Acad Sci. 2001;945:93-102.

11. Schwarzenbach H, Hoon DS, Pantel K. Cell-free nucleic acids as biomarkers in cancer patients. Nat Rev Cancer. 2011;11(6):426-37.

12. Elshimali YI, Khaddour H, Sarkissyan M, Wu Y, Vadgama JV. The clinical utilization of circulating cell free DNA in blood of cancer patients. Int J Mol Sci. 2013;14(9):18925-58

13. Brinkmann V, Reichard U, Goosmann C, et al. Neutrophil extracellular traps kill bacteria. Science. 2004;303(5663):1532-5.

14. Kessenbrock K, Krumbholz M, Schönermarck U, et al. Netting neutrophils in autoimmune small-vessel vasculitis. Nat Med. 2009;15(6):623-5.

15. Summers T, Langan RC, Nissan A, et al. Serum-based DNA methylation biomarkers in colorectal cancer: potential for screening and early detection. J Cancer. 2013:4(3):210-6.

16. Jin $H, M a Y$, Shen $Q$, et al. Circulating methylated DNA as biomarkers for cancer detection. In: Dricu A, editor. Methylation - from DNA, RNA and Histones to Diseases and Treatment. Rijeka: InTech; 2013. p. 137-52.

17. deVos T, Tetzner R, Model F, et al. Circulating methylated SEPT9 DNA in plasma is a biomarker for colorectal cancer. Clin Chem. 2009:55(7):1337-46.

18. Holdenrieder S, Dharuman Y, Standop J, et al. Novel serum nucleosomics biomarkers for the detection of colorectal cancer. Anticancer Res. 2014;34(5):2357-62.

19. Bauden M, Pamart D, Ansari D, et al. Circulating nucleosomes as epigenetic biomarkers in pancreatic cancer. Clin Epigenetics. 2015;7:106.

20. Gezer U, Yörüker EE, Keskin M, Kulle CB, Dharuman Y, Holdenrieder S. Histone methylation marks on circulating nucleosomes as novel blood-based biomarker in colorectal cancer. Int J Mol Sci. 2015;16(12):29654-62.

21. R Development Core Team. R: A language and environment for statistical computing. R Foundation for Statistical Computing, Vienna, Austria. URL http://www.R-project.org/. R Found Stat Comput Vienna, Austria. 2015:2015.

22. Locker GY, Hamilton S, Harris J, et al. ASCO 2006 update of recommendations for the use of tumor markers in gastrointestinal cancer. J Clin Oncol. 2006;24(33):5313-27.

\section{Submit your next manuscript to BioMed Central and we will help you at every step:}

- We accept pre-submission inquiries

- Our selector tool helps you to find the most relevant journal

- We provide round the clock customer support

- Convenient online submission

- Thorough peer review

- Inclusion in PubMed and all major indexing services

- Maximum visibility for your research

Submit your manuscript at www.biomedcentral.com/submit 